13

\title{
Моделирование ионного облучения кристаллических и аморфных мишеней - материалов первой стенки токамака-реактора
}

\author{
( Д.С. Мелузова, П.Ю. Бабенко, А.Н. Зиновьев, А.П. Шергин
}

Физико-технический институт им. А.Ф. Иофрфе РАН,

194021 Санкт-Петербург, Россия

e-mail: dmeluzova@gmail.com

Поступило в Редакцию 5 июля 2021 г.

В окончательной редакции 20 августа 2021 г.

Принято к публикации 20 августа 2021 г.

Представлено обобщение результатов серии работ, посвященных моделированию различных процессов, происходящих при атомной бомбардировке кристаллических и аморфных твердых тел. С помощью оригинальных численных кодов рассчитаны коэффициенты отражения, линейные потери энергии и пробеги атомов в твердом теле, каналирование, а также коэффициенты распыления и их зависимости от угла падения бомбардирующей частицы для комбинаций $\mathrm{Be}-\mathrm{W}$ и $\mathrm{Ne}-\mathrm{W}$. Исследовались мишени из Ве-, $\mathrm{W}$-, C-материалов, входящих в состав поверхностей, подвергающихся воздействию плазмы в различных токамаках. Особое внимание уделено комбинациям атом-мишень, по которым отсутствуют надежные экспериментальные данные. Показано значительное влияние используемого потенциала взаимодействия на результаты моделирования. Рассмотренные результаты объединены как общим предметом исследования изучением процессов взаимодействия ионов плазмы с материалами первой стенки токамака-реактора, так и общим методом исследования - использованием разработанного для этой цели оригинального численного кода.

Ключевые слова: рассеяние, ионное распыление, потенциал взаимодействия, энерговыделение.

DOI: 10.21883/JTF.2021.12.51770.204-21

\section{Введение}

В токамаке ИТЭР бериллиевая первая стенка и дивертор из вольфрама будут подвергаться интенсивному облучению потоком нейтральных атомов и ионов, покидающих плазму. Работа ИТЭР планируется на D-Tплазме, поэтому в наших работах в качестве бомбардирующих частиц рассматривались атомы изотопов водорода, а также атомы Не - продукты термоядерной реакции. Помимо бериллия и вольфрама интерес представляет изучение мишени из углерода, так как в некоторых токамаках присутствуют углеродные поверхности, подвергающиеся воздействию плазмы. Очевидно, что явление отражения атомов от поверхностей в токамаке-реакторе будет играть важную роль в достижении ключевых параметров плазмы и определении тепловой нагрузки на материалы. Отражение атомов от материалов токамака-реактора является предметом интенсивных теоретических исследований, авторы которых отмечают острую нехватку экспериментальных данных [1,2], так как экспериментальные данные по коэффициентам отражения от Ве отсутствуют, а для С и W крайне ограничены $[3,4]$. Также отсутствуют достоверные данные о пробегах перечисленных атомов в аморфном вольфраме. Знание пробегов атомов требуется для оценки образования дефектов в конструкционных материалах токамака-реактора и накопления изотопов водорода в материале. Дополнительно- го изучения требует и распыление вольфрама ионами бериллия: экспериментальные данные по распылению отсутствуют, однако, как показывают исследования [5,6], бомбардировка стенки токамака атомами D и T вызывает значительное поступление бериллия в плазму. Атомы Ве в плазме ионизуются, ускоряются и, в свою очередь, вызывают распыление дивертора, что является крайне нежелательным, так как поступление вольфрама в плазму значительно изменяет характеристики разряда $[7,8]$.

Указанные пробелы возможно восполнить с помощью компьютерного моделирования перечисленных процессов, однако для достоверного численного исследования требуется детальный анализ ряда параметров, описывающих взаимодействие атомных частиц с твердым телом.

\section{1. Методы моделирования}

Моделирование проводилось с помощью оригинального кода, в котором реализованы два подхода к описанию движения атома в твердом теле: в зависимости от условий поставленной задачи использовался или метод траекторий, который является частным случаем молекулярной динамики, или приближение парных столкновений (BCA). Одним из основных параметров, выбор которого влияет на результат моделирования рассеяния атома на поверхности, является потенциал взаимодействия 


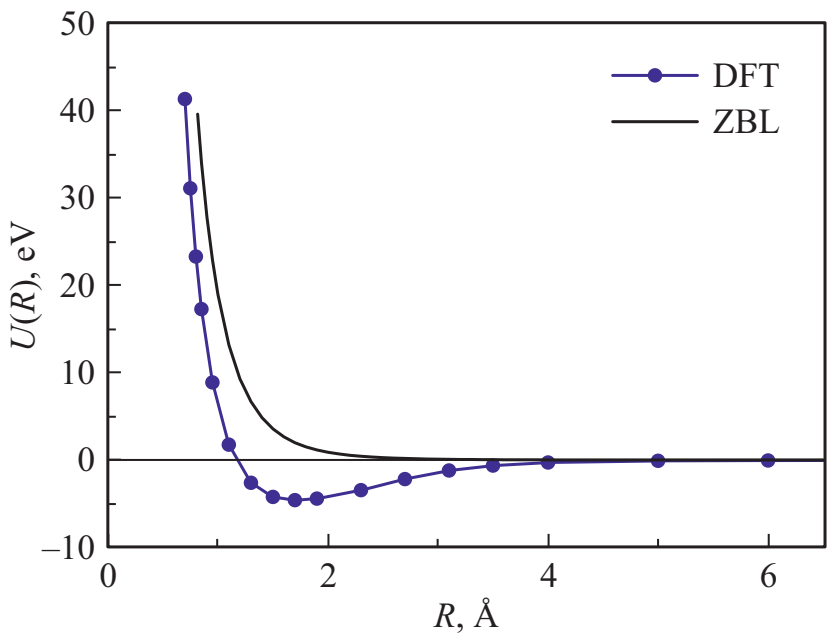

Рис. 1. Сравнение потенциала, полученного методом DFT, с потенциалом ZBL для комбинации D-W.

налетающего атома с атомами мишени. В работе [9] было продемонстрировано, что использование теории функционала плотности (DFT) для определения потенциала взаимодействия атомов дает результат, который лучше согласуется с экспериментальными данными, чем широко используемый потенциал ZBL [10]. Сравнение потенциалов DFT и ZBL представлено на рис. 1. DFTпотенциал предсказывает наличие притягивающей ямы в потенциале взаимодействия, в то время как потенциал ZBL является чисто отталкивательным. С использованием приближения DFT были разработаны оригинальные потенциалы взаимодействия для комбинаций атомов $\mathrm{H}$ и Не с атомами $\mathrm{Be}, \mathrm{C}, \mathrm{W}$ [11]. Положение и глубина потенциальной ямы, полученные с помощью DFT для данных пар, согласуются с экспериментальными и расчетными параметрами соответствующих двухатомных молекул. При моделировании движения атома внутри мишени учитывалось торможение на электронной компоненте твердого тела - для этого были использованы экспериментальные данные из базы данных NDS Международного агентства по атомной энергии [12].

\section{2. Отражение атомов от аморфной поверхности}

В результате моделирования рассеяния атомов $\mathrm{H}$, D и $\mathrm{T}$ на аморфных поверхностях $\mathrm{Be}, \mathrm{W}$ и $\mathrm{C}$ обнаружено неожиданно сильное влияние притягивающей ямы на величину коэффициентов отражения, которое проявляется в случае скользящего падения атомов $\left(<20^{\circ}\right.$ от поверхности) с энергией порядка сотен $\mathrm{eV}$ (рис. 2,a). На рисунке хорошо виден переход от практически стопроцентного отражения, проявляющегося при моделировании с использованием потенциала ZBL, к заметному поглощению частиц поверхностью, которое имеет место при использовании потенциала DFT. При энергиях свыше $2 \mathrm{keV}$ это различие становится незначительным.

Проведение сравнения с экспериментальными данными было возможно только для комбинации D-C (рис. 2,b): продемонстрировано хорошее согласие с результатами эксперимента [4]. Для других комбинаций отсутствуют экспериментальные данные в рассматриваемом диапазоне энергий, однако сравнение с результатами независимых расчетов коэффициентов отражения $[2,13,14]$, которое возможно при энергиях $>1 \mathrm{keV}$, когда выбор потенциала слабо влияет на результат, показало удовлетворительное согласие с полученными значениями. Проведенные проверки дали основание для использования потенциала DFT в расчетах коэффициентов отражения: были рассчитаны коэффициенты отражения атомов $\mathrm{H}, \mathrm{D}, \mathrm{T}, \mathrm{He}$ при бомбардировке аморфных мишеней $\mathrm{Be}, \mathrm{C}, \mathrm{W}[11,15]$ под различными углами в широком энергетическом диапазоне
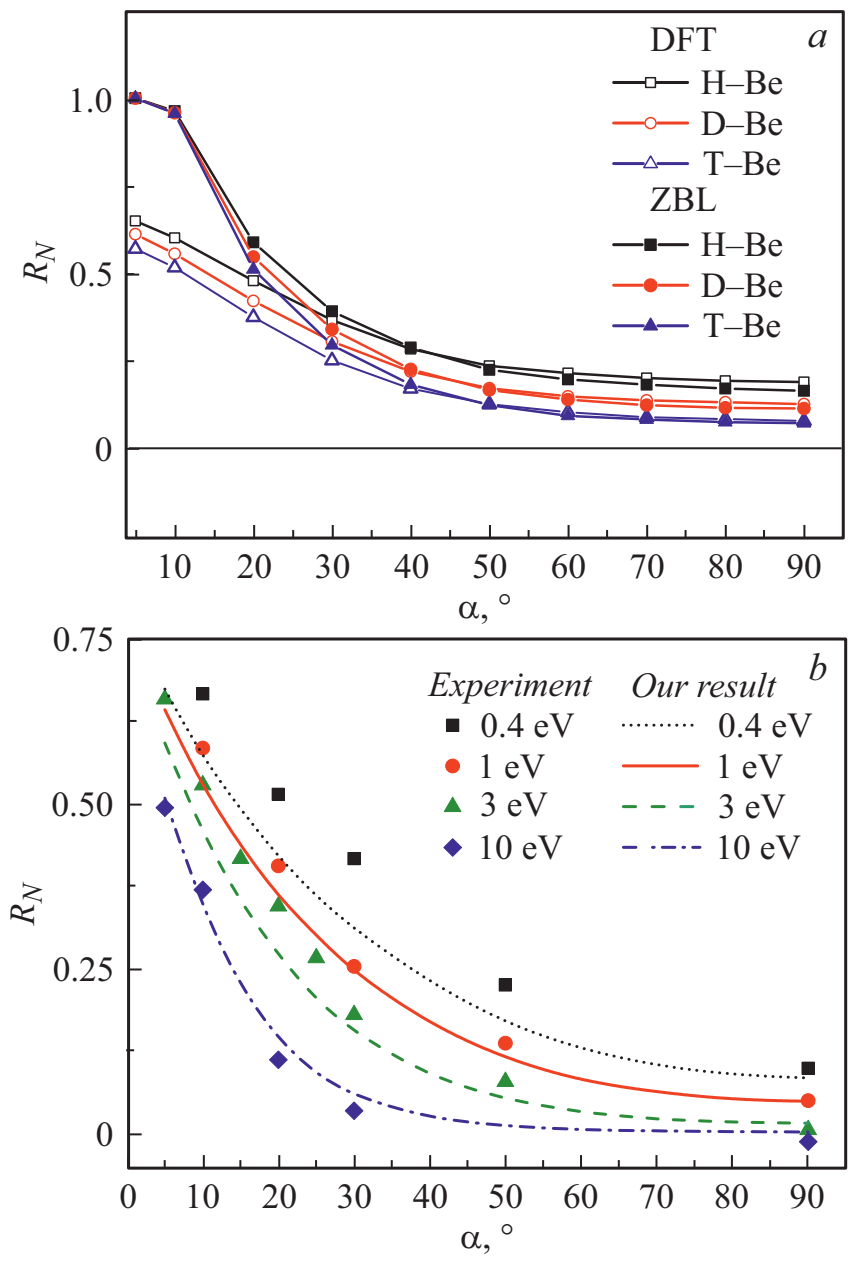

Рис. 2. $a-$ коэффициенты отражения различных изотопов водорода с энергией $100 \mathrm{eV}$ от мишени из Ве в зависимости от угла падения (отсчет от поверхности) при использовании потенциалов DFT и ZBL; $b-$ сравнение коэффициентов отражения, рассчитанных с использованием потенциала DFT, с экспериментальными данными [4] для комбинации D-C. 
$100 \mathrm{eV}-10 \mathrm{keV}$, который соответствует типичным энергиям частиц плазмы.

\section{3. Пробеги атомов в аморфных мишенях}

Влияние притягивающей ямы в потенциале взаимодействия на результаты моделирования было оценено и для моделирования прохождения атомных частиц через аморфное твердое тело. На рис. 3 показано сравнение значений пробегов атомов в вольфраме, рассчитанных с использованием потенциала с ямой и без. Значения пробегов, полученные с использованием потенциала DFT, превышают значения, полученные с потенциалом ZBL, причем различие уменьшается с ростом начальной энергии налетающих частиц. Это можно объяснить тем, что от потенциала взаимодействия зависят ядерные тормозные способности, которые на несколько порядков меньше электронных тормозных способностей. Вклад электронных тормозных способностей в торможение атомной частицы в твердом теле растет при увеличении энергии.

Из-за отсутствия экспериментальных данных о пробегах атомных частиц в вольфраме проверка применимости используемых методов и параметров была выполнена с помощью дополнительного моделирования прохождения атомов водорода через аморфный кремний. Было продемонстрировано [16] хорошее согласие с экспериментальными данными $[17,18]$.

Были рассчитаны распределения пробегов по глубине для комбинаций $\mathrm{H}-\mathrm{Si}$ и $\mathrm{D}-\mathrm{W}$ при начальной энергии бомбардирующих частиц $100 \mathrm{eV}-10 \mathrm{keV}$. Полученные распределения [16] описаны характеристиками, позволяющими построить распределения пробегов по глубине, не прибегая к расчетам.

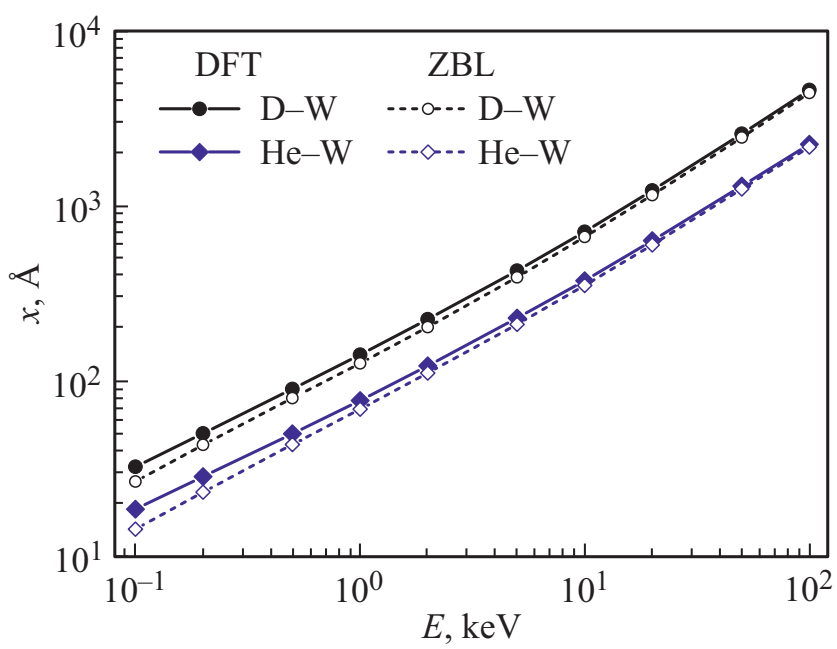

Рис. 3. Сравнение расчетных зависимостей среднего пробега от энергии налетающих частиц для комбинаций $\mathrm{D}-\mathrm{W}, \mathrm{He}-\mathrm{W}$, полученных с использованием потенциалов DFT и ZBL.
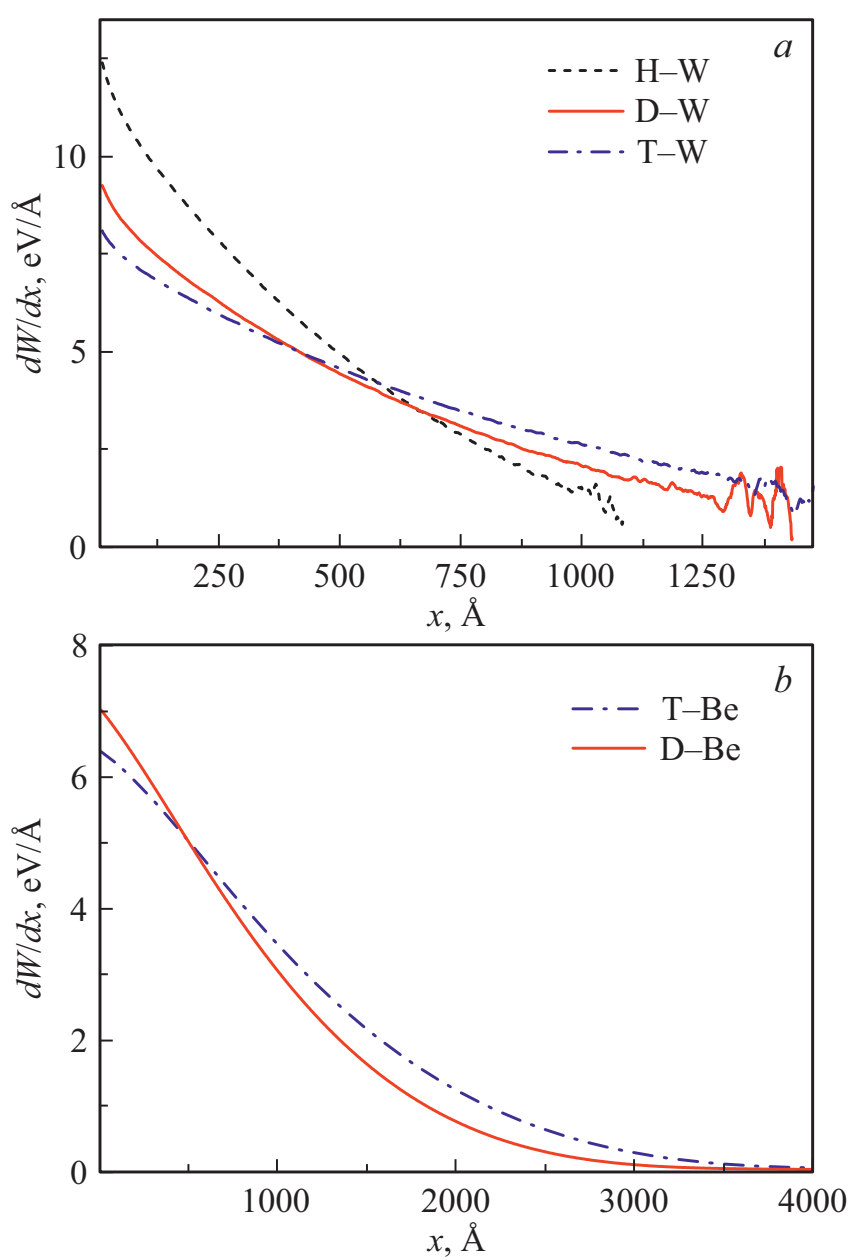

Рис. 4. $a-$ распределение энеговыделения по глубине при прохождении $\mathrm{H}, \mathrm{D}, \mathrm{T}$ с энергией $5 \mathrm{keV}$ через $\mathrm{W} . d W / d x-$ линейные потери энергии (средние потери энергии частицей на интервале $d x) ; b$ - распределение энерговыделения по глубине в Ве, облучаемом типичным для токамака ИТЭР спектром атомов D и T, покидающих плазму, нормированное на одну падающую частицу.

\section{4. Энерговыделение в аморфных мишенях}

Моделирование прохождения атомов H, D, T через аморфные мишени позволило сделать выводы, касающиеся распределения энерговыделения по глубине в материалах токамака-реактора. Показано, что при энергиях налетающих частиц $\lesssim 100 \mathrm{keV}$ максимум энерговыделения приходится на область вблизи поверхности. Динамика изменения характера кривых энерговыделения при дальнейшем увеличении начальной энергии бомбардирующих частиц для комбинации $\mathrm{H}-\mathrm{W}$ показала, что пик Брэгга начинает значительно проявляться только в области энергий $\sim 500 \mathrm{keV}$ [19]. Сравнение распределений энерговыделения по глубине для различных изотопов водорода показало их подобие при одинаковом значении начальной энергии (рис. 4,a). На малой 
глубине энерговыделение в случае Н больше, чем в случае D и T, что можно объяснить большим значением электронных тормозных способностей для более легкого изотопа.

Анализ распределения энерговыделения в материалах первой стенки при облучении пучком частиц с энергиями, соответствующими типичному спектру атомов D и T, покидающих плазму (расчет спектра выполнен кодом DOUBLE-MC), показал [20] (рис. 4,b), что при бомбардировке изучаемых мишеней атомами с угловым и энергетическим распределением, ожидаемым в условиях плазмы токамака ИТЭР, атомы Т проникают в материал примерно на 20\% глубже, чем атомы D, что может приводить к опасному накоплению трития в первой стенке.

\section{5. Прохождение атомов через кристалл - режим каналирования}

Помимо исследования прохождения атомов через аморфные мишени также было проведено моделирование явления каналирования в кристаллах. Были рассчитаны распределения пробегов атомов D в W(100) при различных начальных энергиях [21] и углах падения. Рис. 5 иллюстрирует, как изменяется доля частиц, захваченных в канал, при изменении угла падения пучка на несколько градусов относительно нормали: с ростом угла распределение пробегов трансформируется, отражая уменьшение доли частиц, захваченных в канал. Видно, что при отклонении на $6^{\circ}$ доля частиц в канале составляет $\sim 1 \%$. Данный результат находится в согласии с теоретической оценкой критического угла каналирования в вольфраме [22].

Проведен анализ эволюции пространственного распределения пучка атомов D, захваченных в канал кристалла W(100). Для этого было выполнено модели-

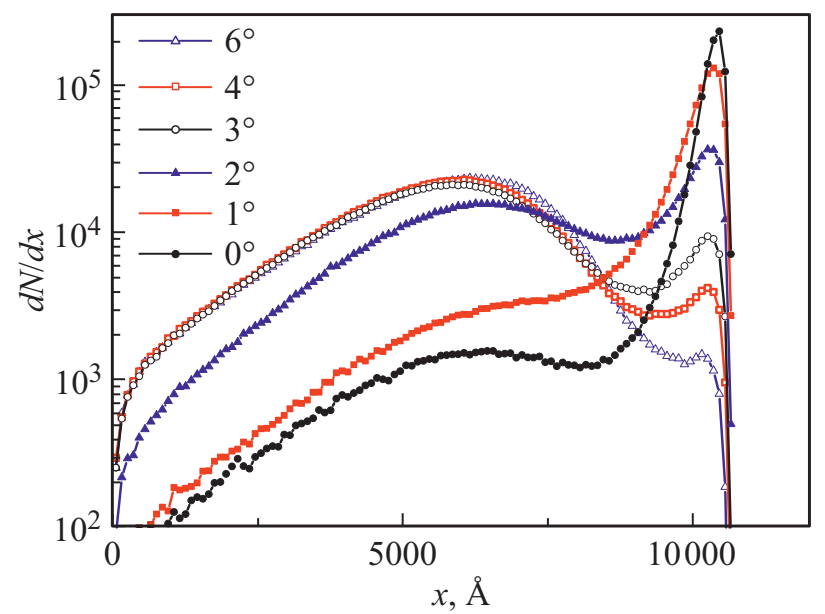

Рис. 5. Распределения пробегов по глубине материала для комбинации $\mathrm{D}-\mathrm{W}(100)$ при различных углах падения относительно нормали к поверхности; энергия налетающих частиц $100 \mathrm{keV}$ рование прохождения атомов D, бомбардирующих поверхность в пределах одной ячейки кристаллической решетки, через мишень W(100). На глубине 1000, 3000, 7000 и $9000 \AA$ фиксировалось пространственное распределение частиц в облучаемом канале и в соседних каналах. На рис. 6 продемонстрирована динамика изменения распределения атомных частиц в канале. Обнаружено образование четкой структуры в пространственном распределении частиц и ее сохранение вплоть до глубины, составляющей до $90 \%$ от пробега частиц. Форма сформированной структуры находится в согласии с независимым аналитическим предсказанием [23] и определяется распределением суммарного потенциала в канале, что позволяет предложить эксперимент по его определению из углового распределения частиц, вылетевших из кристалла после прохождения 30-60\% от глубины проникновения.

\section{6. Распыление аморфной мишени}

Для изучения распыления вольфрама ионами бериллия был разработан код, основанный на принципах молекулярной динамики (MD) и оптимизированный под специфику поставленной задачи. Мишень была представлена как аморфное твердое тело, взаимодействие между ее атомами было описано с помощью многочастичного потенциала в рамках модели погруженного атома [24]. Из-за отсутствия экспериментальных данных по коэффициентам распыления для комбинации $\mathrm{Be}-\mathrm{W}$ было проведено дополнительное моделирование для комбинации $\mathrm{Ne}-\mathrm{W}$. Для обеих комбинаций были получены зависимости коэффициента отражения от энергии налетающих частиц (рис. 7) и от угла падения. В случае распыления вольфрама ионами неона продемонстрировано согласие с экспериментальными данными [25]. Для сравнения представлены результаты расчета широко используемой программой SRIM [26] — видно, что она завышает величину порога распыления. Для комбинации $\mathrm{Be}-\mathrm{W}$ было достигнуто согласие с молекулярно-динамическим расчетом программой LAMMPS, приведенным в работе [27].

Нами предложена модель, позволяющая рассчитать коэффициенты распыления вольфрама легкими ионами, бомбардирующими мишень. Для применения данной модели необходимо знать энергетическое и угловое распределение обратнорассеянных первичных частиц, бомбардирующих приповерхностные слои мишени. По формуле

$$
Q=\frac{4 M_{1} M_{2}}{\left(M_{1}+M_{2}\right)^{2}} \cdot E_{1} \cdot \sin ^{2}\left(\frac{\chi}{2}\right)
$$

определяется энергия, переданная ионом с массой $M_{1}$ и энергией $E_{1}$ покоящемуся атому мишени с массой $M_{2}$, который принадлежит поверхностному слою. $\chi-$ угол рассеяния в системе центра масс. Условие распыления 

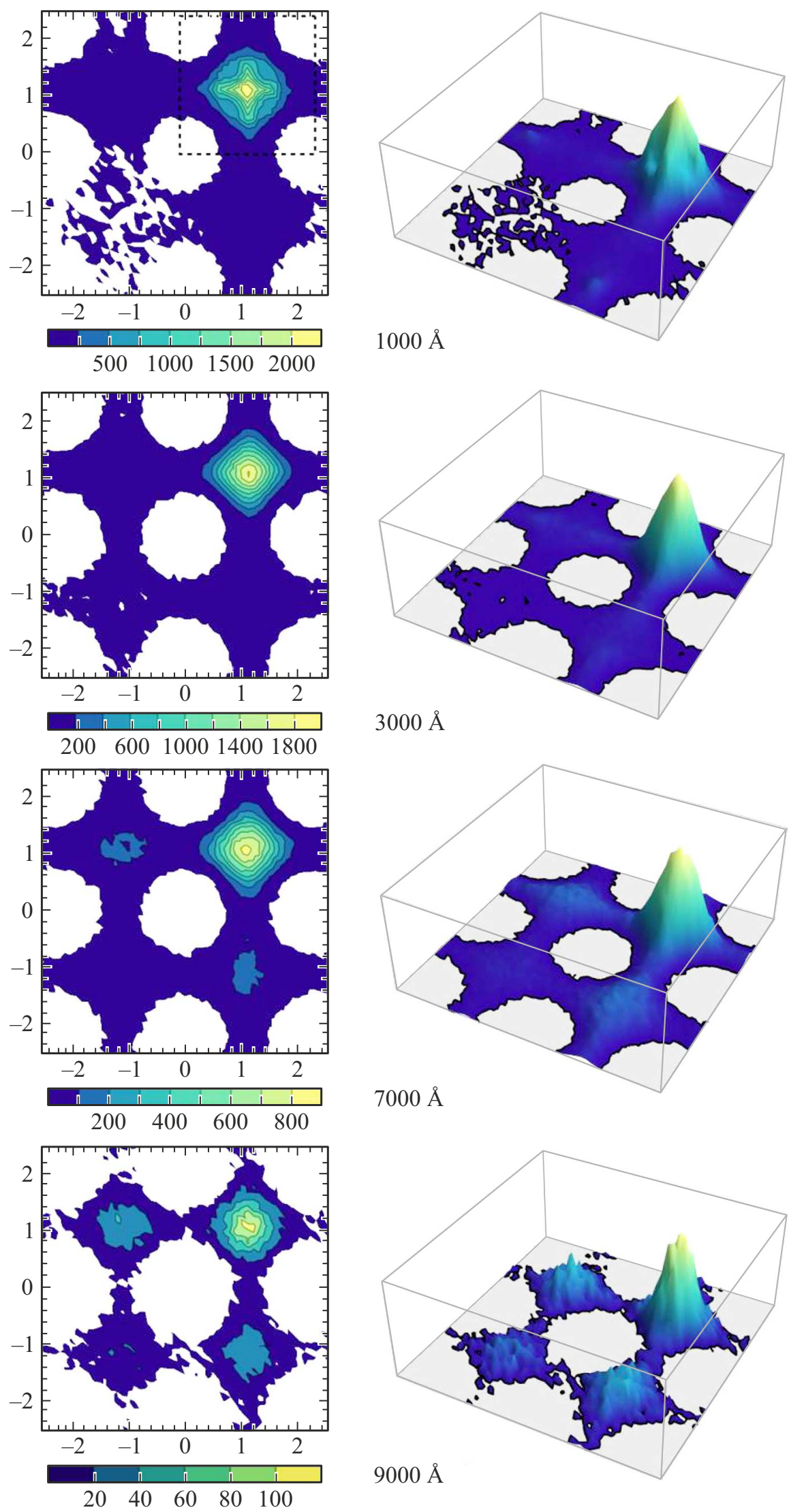

Рис. 6. Пространственное распределение частиц, захваченных в канал, на глубине 1000, 3000, 7000 и $9000 \AA$ для комбинации $\mathrm{D}-\mathrm{W}(100)$, начальная энергия $100 \mathrm{keV}$. На осях расстояние в А. Цветовая шкала показывает количество частиц, зарегистрированных в каждой точке пространства. Пунктирный квадрат - область облучения пучком $D$, соответствующая одному каналу кристалла. 
определяется следующим образом: компонента энергии $Q$, переданная атому вольфрама вдоль оси, перпендикулярной поверхности, должна превышать энергию сублимации, которая для вольфрама равна $8.45 \mathrm{eV}$. В соответствии с данным условием определяются пороговые значения $E_{1}$ и $\chi\left(E_{t h}\right.$ и $\chi_{t h}$ соответственно), при которых реализуется распыление поверхностного слоя мишени. Из данных по обратному рассеянию ионов отбирается число случаев, когда при соударении атому материала перпендикулярно поверхности передается энергия, превышающая энергию сублимации. Поделив число распыленных атомов на число первоначально падающих частиц, можно определить коэффициент распыления. Результат, полученный с использованием данной модели, показан на рис. 7 (BSS - Back Scattering Sputtering). Видно, что расчет с помощью нашей модели согласу-
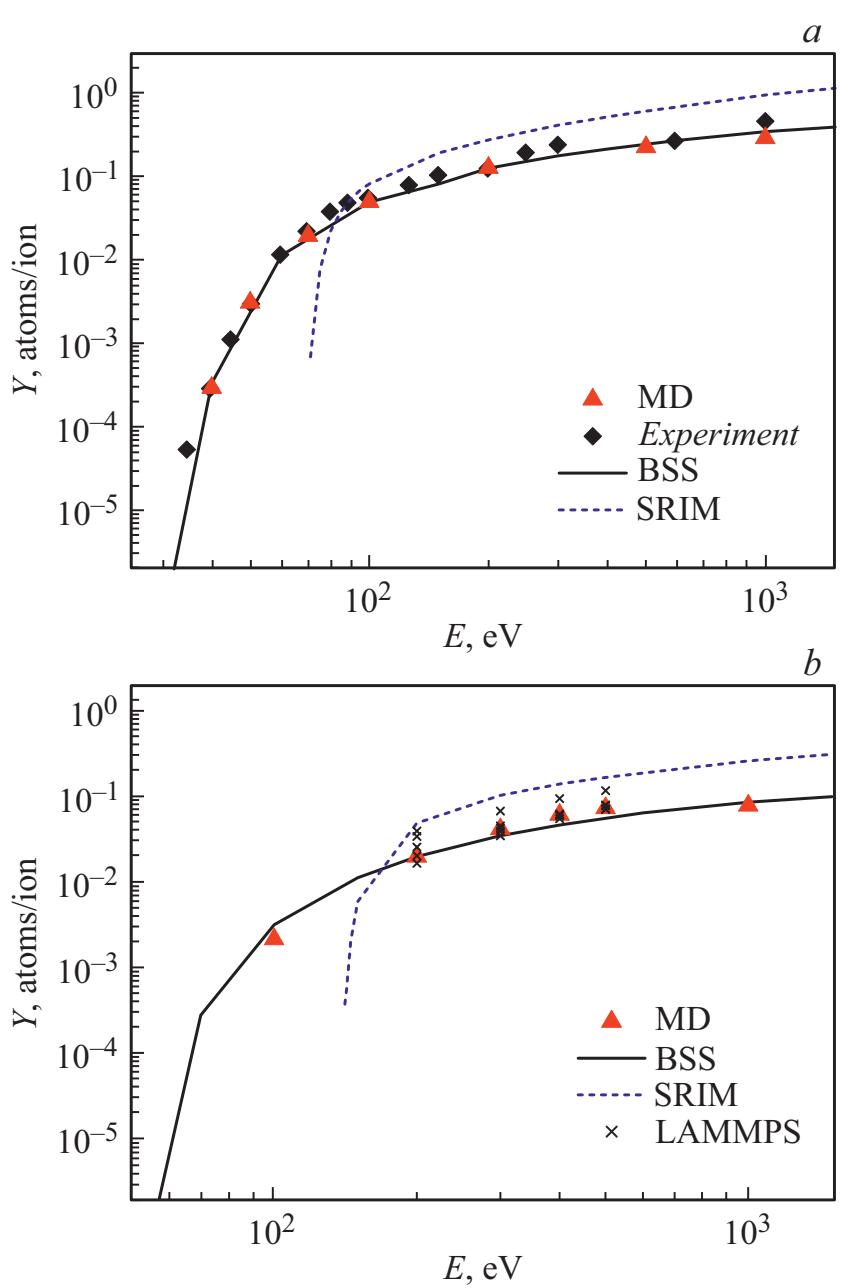

Рис. 7. $a-$ зависимость коэффициента распыления $\mathrm{Y}$ от энергии налетающих частиц для комбинации $\mathrm{Ne}-\mathrm{W}$ : наш молекулярно-динамический расчет, расчет с помощью модели BSS, экспериментальные данные из работы [25] и расчет программой SRIM [26]; $b-$ зависимость коэффициента распыления Y от энергии налетающих частиц для комбинации $\mathrm{Be}-\mathrm{W}$ : наш молекулярно-динамический расчет, расчет с помощью модели BSS, расчет LAMMPS из работы [27] и расчет программой SRIM [26].

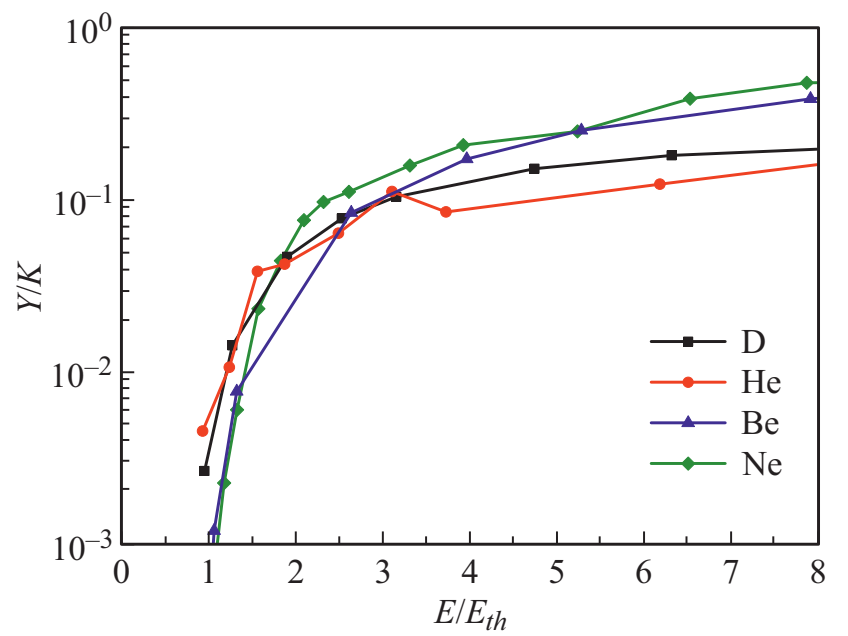

Рис. 8. Зависимость коэффициента распыления W ионами D, $\mathrm{He}, \mathrm{Be}, \mathrm{Ne}$ в приведенных координатах. $K=\sigma\left(\chi_{t h}\right) / d^{2}$ при $E / E_{t h}=4$, где $d-$ среднее расстояние между слоями $\mathrm{W}$.

ется с молекулярно-динамическим расчетом, а также с экспериментальными данными для $\mathrm{Ne}-\mathrm{W}$.

На рис. 8 в приведенных координатах представлены коэффициенты распыления вольфрама ионами $\mathrm{D}$, Не, $\mathrm{Be}, \mathrm{Ne}$, полученные с использованием разработанной модели. Наша модель демонстрирует универсальность зависимостей коэффициентов распыления от энергии в припороговой области. Видно, что кривые для ионов, близких по массе, близки между собой. Имеется возможность экстраполировать результаты на неизученные случаи.

\section{Заключение}

Основные результаты проведенных работ состоят в следующем:

1. Разработан численный код для моделирования взаимодействия пучков ионов и атомов с твердым телом. Сопоставление получаемых результатов с экспериментом для процессов отражения частиц, пробегов частиц в кристаллических и аморфных мишенях, распыления при ионной бомбардировке позволяет подтвердить надежность получаемых данных.

2. Получены коэффициенты отражения для всех комбинаций атомов $\mathrm{H}, \mathrm{D}, \mathrm{T}, \mathrm{He}$ и аморфных поверхностей $\mathrm{Be}, \mathrm{C}, \mathrm{W}$ в диапазоне энергий от $100 \mathrm{eV}$ до $10 \mathrm{keV}$ при различных углах падения налетающих частиц. Обнаружено неожиданно сильное влияние притягивающей ямы в потенциале на результат при энергиях порядка сотен еV.

3. Рассчитаны пробеги атомов H, D, Не в аморфных $\mathrm{Si}$ и W. Предложены формулы для описания полученных результатов.

4. В результате моделирования явления каналирования в кристаллических мишенях рассчитаны пробеги 
атомов $\mathrm{H}$ и $\mathrm{D}$ в $\mathrm{W}(100)$ и показано изменение характера распределения пробегов по глубине в зависимости от энергии атомов. Проведен анализ эволюции пространственного распределения каналируемого пучка в W(100), который показал образование четкой пространственной структуры каналируемого пучка, coxраняющейся на 90\% пути частиц в канале.

5. Показано преобладание энерговыделения вблизи поверхности мишени при бомбардировке аморфных поверхностей $\mathrm{Be}, \mathrm{C}, \mathrm{W}$ изотопами водорода с энергиями до $100 \mathrm{keV}$. На основе полученных распределений рассчитано энерговыделение на одну падающую частицу при облучении указанных материалов атомами с энергетическим спектром, характерным для токамака ИТЭР. Предсказано накопление трития в первой стенке токамака-реактора.

6. Рассчитаны зависимости коэффициентов распыления $\mathrm{W}$ ионами $\mathrm{Ne}$ и Ве от энергии и угла падения налетающих частиц. Разработана модель распыления вольфрама обратно рассеянными легкими ионами, объясняющая универсальный характер зависимостей коэффициентов распыления от энергии в припороговой области.

Как показано в работе, применение компьютерного моделирования в сочетании с уточнением наших представлений о потенциалах взаимодействия частиц позволяет успешно устранить пробелы в случаях, когда эксперимент отсутствует. Полученные данные имеют существенное значение для моделирования различных процессов в пристеночной плазме токамака-реактора.

\section{Конфликт интересов}

Авторы заявляют, что у них нет конфликта интересов.

\section{Список литературы}

[1] V. Borovikov, A.F. Voter, X.-Z. Tang. J. Nucl. Mater., 447 (1), 254 (2014). DOI: 1016/j.jnucmat.2014.01.021

[2] P.N. Maya. J. Nucl. Mater., 480, 411 (2016). DOI: 10.1016/j.jnucmat.2016.08.007

[3] V.V. Bandurko, N.N. Koborov, V.A. Kurnaev, V.M. Sotnikov, O.V. Zabeyda. J. Nucl. Mater., 176-177, 630 (1990). DOI: 10.1016/0022-3115(90)90118-7

[4] C.K. Chen, B.M.U. Scherzer, W. Eckstein, Appl. Phys. A, 33, 265 (1984).

[5] P.Yu. Babenko, M.I. Mironov, V.S. Mikhailov, A.N. Zinoviev. Plasma Phys. Control. Fusion, 62, 045020 (2020). DOI: $10.1088 / 1361-6587 / a b 7943$

[6] S. Makarov, E. Kaveeva. MATEC Web Conf. EECE-2018, 245, 13002 (2018). DOI: 10.1051/matecconf/201824513002

[7] F. Kö chl, A. Loarte, E. de la Luna, V. Parail, G. Corrigan, D. Harting, I. Nunes, C. Reux, F.G. Rimini, A. Polevoi, M. Romanelli and JET Contributors. Plasma Phys. Control. Fusion, 60, 074008 (2018). DOI: 10.1088/1361-6587/aabf52

[8] T. Abrams, E.A. Unterberg, D.L. Rudakov, A.W. Leonard, O. Schmitz, D. Shiraki, L.R. Baylor, P.C. Stangeby, D.M. Thomas, H.Q. Wang. Phys. Plasmas, 26, 062504 (2019). DOI: $10.1063 / 1.5089895$
[9] A.N. Zinoviev, K. Nordlund. Nucl. Instrum. Methods Phys. Res. B, 406, 511 (2017). DOI: 10.1016/j.nimb.2017.03.047

[10] J.F. Ziegler, J.P. Biersack, U. Littmark. The Stopping and Range of Ions in Solids, (Pergamon, NY., 1985)

[11] D.S. Meluzova, P.Yu. Babenko, A.P. Shergin, K. Nordlund, A.N. Zinoviev. Nucl. Instrum. Methods Phys. Res. B, 460, 4 (2019). DOI: 10.1016/j.nimb.2019.03.037

[12] H. Paul. IAEA NDS. https://www-nds.iaea.org/stopping

[13] A. Lasa, C. Björkas, K. Vö rtler, K. Nordlund. J. Nucl. Mater., 429, 284 (2012). DOI: 10.1016/j.jnucmat.2012.06.012

[14] Д.К. Когут, Н.Н. Трифонов, В.А. Курнаев. Изв. РАН. Сер. физ., 72 (7), 1024 (2008) [D.K. Kogut, N.N. Trifonov, V.A. Kurnaev. Bulletin of the Russian Academy of Sciences: Physics, 72 (7), 969 (2008).]

[15] Д.С. Мелузова, П.Ю. Бабенко, А.П. Шергин, А.Н. Зиновьев. Поверхность, 7, 98 (2020). DOI: 10.31857/S1028096020070146 [D.S. Meluzova, P.Yu. Babenko, A.P. Shergin, A.N. Zinoviev. J. Surf. Invest., 14, 738 (2020). DOI: 10.1134/S102745102004014X]

[16] Д.С. Мелузова, П.Ю. Бабенко, А.П. Шергин, А.Н. Зиновьев. ЖТФ, 90 (1), 155 (2020).

DOI: $10.21883 /$ JTF.2020.01.48678.89-19 [D.S. Meluzova, P.Yu. Babenko, A.P. Shergin, A.N. Zinoviev. Tech. Phys., 65 (1), 145 (2020). DOI: 10.1134/S106378422001017X]

[17] E. Ligeon, A. Guivarc'h. Rad. Eff., 27 (3-4), 129 (1976). DOI: $10.1080 / 00337577608243025$

[18] M. Weiser, M. Behar, S. Kalbitzer, P. Oberschachtsiek, D. Fink, G. Frech. Nucl. Instrum. Methods Phys. Res. B, 29, 487 (1987). DOI: 10.1016/0168-583X(87)90073-5

[19] Д.С. Мелузова, П.Ю. Бабенко, М.И. Миронов, В.С. Михайлов, А.П. Шергин, А.Н. Зиновьев. Письма в ЖТФ, 45 (11), 51 (2019). DOI: 10.21883/PJTF.2019.11.47827.17771 [D.S. Meluzova, P.Yu. Babenko, M.I. Mironov, V.S. Mikhailov, A.P. Shergin, A.N. Zinoviev. Tech. Phys. Lett., 45 (6), 580 (2019). DOI: 10.1134/S1063785019060130]

[20] D.S. Meluzova, P.Yu. Babenko, M.I. Mironov, V.S. Mikhailov, A.P. Shergin, A.N. Zinoviev. AIP Conf. Proc., 2179, 020018 (2019). DOI: $10.1063 / 1.5135491$

[21] Д.С. Мелузова, П.Ю. Бабенко, А.П. Шергин, А.Н. Зиновьев. Письма в ЖТФ, 46 (5), 34 (2020).

DOI: 10.21883/PJTF.2020.05.49106.18034 [D.S. Meluzova, P.Yu. Babenko, A.P. Shergin, A.N. Zinoviev. Tech. Phys. Lett., 46 (3), 235 (2020). DOI: 10.1134/S1063785020030104]

[22] С.И. Матюхин. ЖТФ, 78 (12), 47 (2008). [S.I. Matyukhin. Tech. Phys., 53 (12), 1578 (2008). DOI: $10.1134 / \mathrm{S} 1063784208120074]$

[23] L.C. Feldman, J.W. Mayer, S.T. Picraux. Materials Analysis by Ion Channeling (Academic Press, San Diego, 1982)

[24] M.-C. Marinica, L. Ventelon, M.R. Gilbert, L. Proville, S.L. Dudarev, J. Marian, G. Bencteux, F. Willaime. J. Phys.: Condens. Matter., 25, 395502 (2013). DOI: $10.1088 / 0953-8984 / 25 / 39 / 395502$

[25] R. Behrisch, W. Eckstein. Sputtering by Particle Bombardment (Springer, Berlin, 2007)

[26] J.F. Ziegler, J.P. Biersack. SRIM. http://www.srim.org

[27] X. Yang, A. Hassanein. Appl. Surf. Sci., 293, 187 (2014). DOI: $10.1016 /$ j.apsusc.2013.12.129 